MPI-PhT 2001-??

\title{
Modifying operator product expansions by non-perturbative non-locality
}

\author{
Ralf Hofmann \\ Max-Planck-Institut für Physik \\ Werner-Heisenberg-Institut \\ Föhringer Ring 6, 80805 München \\ Germany
}

\begin{abstract}
Local quark-hadron duality violations in conventional applications of the operator product expansion are proposed to have their origin in the fact that the QCD vacuum or a hadronic state is not only characterized by nonvanishing expectation values of local, gauge invariant operators but also by finite correlation lengths of the corresponding gauge invariant $n$-point functions. Utilizing high-resolution lattice information on these correlators a non-perturbative component of OPE coarse graining is proposed which, in principle, allows for a determination of the critical dimension where the break-down of the expansion sets in.
\end{abstract}


A practical and very successful approach to relate hadronic parameters like masses, decay constants and widths to a few universal parameters of the vacuum of Quantum Chromodynamics [1] was introduced a long time ago [2]. The method of QCD sum rules (QSR's) relies on Wilson's operator product expansion (OPE) [3] at an external, euclidean momentum $Q$, analyticity of the considered QCD current correlator everywhere in the plane except for a cut along the positive real axis, and perturbative calculability of the operator coefficients. non-perturbative effects are introduced as power corrections via the nonvanishing vacuum averages of local, gauge invariant operators. If the average of an operator of mass dimension $d$ is comparable with $\Lambda^{d}$, where $\Lambda$ is a typical hadronic scale then modulo numerical factors power corrections are suppressed by $(\Lambda / Q)^{d}$, and one may hope that the expansion approximates the correlator at least in an asymptotic sense [4]. To make contact with hadronic properties one assumes quark-hadron duality, namely the property of the dispersive part of the correlator to be described in terms of measured hadronic cross sections. By appropriately adjusting the external momentum QSR's relate the properties of the lowest resonance to the non-perturbative condensates. There is not much doubt that averaged spectral functions relevant in QSR's are dual to the OPE [41. In practice it is fair to say, however, that there are channels where the sum rule method seems to be jeopardized by abnormally large non-perturbative corrections (scalar, pseudoscalar).

However, it is painfully obvious that the conventional, practical OPE, which is typically truncated at dimension $d=6$ or $d=8$ does not account for pointwise or local duality (for a summary see the review articles [4, 5]). Usually the asymptotic nature of the OPE is blamed for this. Indeed, perturbatively calculated Wilson coefficients being of the form $\left(\alpha_{s}\left(Q_{0}\right) /\left(\alpha_{s}(Q)\right)^{\gamma} \times\left(Q^{2}\right)^{-d}\right.$, where $\gamma$ denotes an (effective) anomalous dimension and $Q_{0}$ is a normalization scale, do not allow for the observed "wiggling" of the spectral function obtained by taking the imaginary parts of a term-by-term continuation to time-like momenta. Since the expansion is truncated terms, which behave like $\exp \left[-\right.$ const $\left.\sqrt{Q^{2} / \Lambda^{2}}\right]$, can not be captured [5]. On the other hand, such terms contribute asymptotically unsuppressed oscillations to the spectral function which contradicts experiment and is not allowed by asymptotic freedom. Investigations concerning the origin of local duality violation have been performed within various models in the literature [6, 7, 8]. Impressively, it was shown in [6, 7] that upon appealing to a dispersion relation a model spectral function with equally spaced narrow resonances yields an asymptotic expansion in the euclidean which resembles a conventional OPE with factorially growing coefficients. However, such a model ignores the drastic broadening of resonances and the decrease of their heights with increasing energy.

Local duality violations may have a considerable impact on the calculation of life-time differences and inclusive/exclusive decay widths of $B$-mesons where OPE in conjunction with the heavy quark expansion is applied at the large time-like momentum $p \sim m_{b}$ [9, 10]. The precise theoretical determination of these quantities is of particularily acute relevance since it would lead to stringent constraints on the 
parameters of the underlying model (e.g. CKM matrix elements in the Standard Model (SM) and/or couplings, masses, and CP violating phases of SM extensions). This, in turn, is of paramount importance for the potential detection of New Physics [11. It is therefore essential to improve our understanding of local duality and to estimate the scales which govern it.

The purpose of this letter is to point out and exploit a genuine source for OPE modification which has only been vaguely addressed so far [5]. The observation is that the conventional OPE of a current-current correlator, which can be proven to exist perturbatively, does not account for the diminishing relevance of local operators composed of the fundamental fields in an asymptotically free theory like QCD when decreasing the spacetime resolution [12]. This resolution, however, is determined by the external (euclidean) momentum squared $Q^{2}=-q^{2}$. The physical picture behind this can be examplified in the case of a correlator of light quark currents as follows [2]: An external current creates a quark-antiquark pair with total momentum $q$. Due to asymptotic freedom the propagation of these degrees of freedom is dominated by perturbation theory at high enough $Q \equiv \sqrt{Q^{2}}$ and comparable momenta. non-perturbative effects become important if there are soft emissions in the process, which are effectively described by nonvanishing vacuum expectation values (VEV's) of gauge invariant, local operators. But what does local really mean? We believe that the answer to this question is tightly connected to the issue of pointwise duality. During the time interval between emission and reabsorption of the constituent degrees of freedom of a hadron the vacuum, which admits these propagations, is being resolved precisely by the external momentum $Q$. So if the OPE is an expansion involving local operators one also ought to say how resolved the corresponding locality is. The renormalization group teaches that information on the VEV of some local operator at a given resolution derives from local and non-local information at higher resolution. In perturbation theory, where interactions do not generate effective fields and parameters out of their fundamental counterparts, this is done by normalizing the corresponding operator at a definite momentum scale $Q_{0}$ and relating this to the momentum $Q$ of the process by means of the corresponding set of renormalization group equations. Since in QSR's the VEV of a local operator is supposed to exclusively carry non-perturbative information one must apply an exclusively non-perturbative procedure of coarse graining to it. The key to non-perturbative coarse graining for the lowest dimensional operators is information on the corresponding gauge invariant 2-point functions [13] which is available from lattice calculations [14]. For higher dimensional operators one so far has to appropriately impose vacuum saturation to express their correlators in terms of the 2-point functions.

The idea that due to the existence of finite correlation lengths in gauge invariant 2-point functions non-local, non-perturbative effects should be included in the $\mathrm{OPE}$ is not new. We just mention here that to obtain realistic hadronic wave functions along the lines of ref. [16] non-local effects were systematically included via an expansion in covariant derivatives in ref. [17]. On the other hand, based on pertur- 
bative arguments it was shown in refs. 18 that a light-cone expansion of the product of gauge invariant currents can be written in terms of non-local string operators. Working in position space, perturbative evolution equations were derived for these operators in 19]. In this paper we introduce non-perturbative, non-locality within a phenomenological generalization of the standard Wilson OPE. Although we concentrate on vacuum correlators it is clear that the procedure is extendable to hadronic state averages. Applications of the concept outlined above to OPE's and spectral functions of various light quark channels are reported in ref. [20].

We start out by expanding the correlator into a conventional OPE at a large external, euclidean momentum $Q$, where we expect the description of the dynamics in terms of the continuum action and local operators made of fundamental fields to be sufficiently accurate. To proceed to lower external momenta Wilson coefficients are evolved perturbatively by means of the running coupling $\alpha_{s}\left(Q^{2}\right)$ and the anomalous dimensions of the operators. The evolution of an operator average can be obtained using information on the non-perturbative part of the corresponding gauge invariant correlator in euclidean position space

$$
\langle F(0)\rangle_{Q}^{n p} \equiv\left\langle F_{1}(0) \cdots F_{n}(0)\right\rangle_{Q}^{n p} \rightarrow 1 / \mathcal{N} \sum\left\langle F_{1}(0) \cdots F_{n}\left(x_{n}\right)\right\rangle_{Q}^{n p}
$$

Thereby, appropriate parallel transporters

$$
S(0, x) \equiv \mathcal{P} \exp \left[i g \int_{0}^{x} d z_{\mu} A_{\mu}\right]
$$

in the non-local expression are understood to make the object gauge invariant. The sum runs over all possible (piecewise straight [13]) trajectories of parallel transport. A normalization $1 / \mathcal{N}$ depending on the mass dimension $d$ and the numbers of fields transforming under the fundamental and adjoint representation is implied to reduce the correlation function to the "condensate" in the limit $x_{1}, \cdots, x_{n} \rightarrow 0$. As examples we give the following dimension 5 and 6 operator VEV's

$$
(i)\left\langle\bar{\psi}(0) F_{\mu \nu}(0) \sigma_{\mu \nu} \psi(0)\right\rangle_{Q}^{n p}, \quad \quad(i i)\left\langle\operatorname{tr} F_{\mu \nu}(0) F_{\nu \kappa}(0) F_{\kappa \mu}(0)\right\rangle_{Q}^{n p}
$$

If an arrow pointing from $x_{i}$ towards $x_{j}$ stands for the parallel transport $S\left(x_{i}, x_{j}\right)$ then the relevant contribution to the correlators correponding to the local operators of eq. (3) can be depicted as in Fig. 1. Note that points with fields transforming under the fundamental (adjoint) representation are connected to one (two) lines of parallel transport.

To coarse grain from resolution $Q$ to resolution $Q-d Q$ we propose to average the non-perturbative part of a correlator corresponding to a connected diagrams over a (euclidean) ball of radius $d R_{Q}$ with

$$
d R_{Q}=\frac{1}{Q-d Q}-\frac{1}{Q}=\frac{1}{Q}\left(\frac{1}{1-\frac{d Q}{Q}}-1\right) \sim \frac{d Q}{Q^{2}}
$$


Figure 1: Diagrammatic representation of the gauge invariant correlators corresponding to the local operators of $(i)$ and $(i i)$ in eq. (3).

In the short-hand notation of eq. (11) this is written as

$$
\langle F(0)\rangle_{Q-d Q}^{n p}=\frac{1}{\left(V\left(d R_{Q}\right)\right)^{(n-1)}} \int_{\left|x_{1}\right|, \cdots,\left|x_{n}\right| \leq d R_{Q}} d^{4} x_{1} \cdots d^{4} x_{n}\left\langle F_{1}(0) \cdots F_{n}\left(x_{n}\right)\right\rangle_{Q}^{n p}
$$

where

$$
V\left(d R_{Q}\right)=\frac{1}{2} \pi^{2}\left(d R_{Q}\right)^{4}=\frac{1}{2} \pi^{2}\left(\frac{d Q}{Q^{2}}\right)^{4}
$$

As for correlators described by disconnected diagrams the application of the factorization hypothesis (vacuum saturation) is discussed in [20] for the example of 4-quark operators composed of color singlet or octet currents.

Let us now concentrate on 2-point functions as they are relevant for dimension 3 and 4 quark and gluon operators, respectively. To our knowledge only the gauge invariant bilocal quark correlators (vector and scalar) [14] and the gluonic field strength correlator 13 have been measured on the lattice. The results suggest that there exists an additive decomposition into a perturbative, power-like in $|x|$, and a non-perturbative, exponential in $|x|$ piece 14 . We are interested in the latter

$$
\left\langle F_{1}(0) F_{2}(x)\right\rangle_{Q}^{n p}=A(Q) \exp (-|x| / \lambda) .
$$

Since $\lambda$ is expected to have direct physical meaning - see for example ref. [21], where in the case of the field strength correlator $\lambda^{-1}$ was interpreted as the energy difference $\bar{\Lambda}$ between a heavy hybrid (an adjoint quark with glue) and the mass of the heavy quark - it can not depend on the resolution $Q$. So coarse graining may only affect the pre-exponential factor $A(Q)$. To derive an evolution equation for $A(Q)$ we combine eqs. (可) and (17):

$$
\left(V\left(d Q / Q^{2}\right)\right)^{-1} \int_{|x| \leq \frac{d Q}{Q^{2}}} d^{4} x A(Q) \mathrm{e}^{-|x| / \lambda}=A(Q-d Q) \mathrm{e}^{-0 / \lambda}=A(Q-d Q)
$$

\footnotetext{
${ }^{1}$ There have been a number of attempts to calculate gauge invariant quark and gluon correlators in the instanton vacuum [15]. In this work, however, we do restrict ourselves to direct lattice results.
} 
Note that in the limit $\lambda \rightarrow \infty$ this leaves $A$ invariant. In this limit we recover the conventional treatment of non-perturbative corrections in the framework of the OPE. Expanding the l.h.s. and r.h.s. of eq. (8) in $\frac{d Q}{Q^{2}}$ and comparing coefficients of the linear terms, we obtain

$$
\frac{d}{d Q} A(Q)=\frac{4}{5 \lambda} Q^{-2} A(Q)
$$

Comparison of zero order terms yields an identity as it should. Evolution equations like eq. (9) could be derived for $(n>2)$-point functions if the explicit form of the non-perturbative part could be extracted from the lattice. The solution of (9) is

$$
A(Q)=A\left(Q_{0}\right) \exp \left[-\frac{4}{5 \lambda}\left(\frac{1}{Q}-\frac{1}{Q_{0}}\right)\right] \text {. }
$$

Disregarding powers of logarithms in the Wilson coefficients, which come from the anomalous dimensions of the corresponding operators and combined with the running coupling $\alpha_{s}\left(Q^{2}\right)$ possess exponents which are numerically small for the naively vacuum saturated 4-quark operators in the OPE's of light-quark correlators [2], eq. (10) implies the following, generic form for the non-perturbative corrections of dimension 4 and 6

$$
\frac{A_{4}\left(Q_{0}\right)}{\left(Q^{2}\right)^{2}} \exp \left[-\frac{4}{5 \lambda_{4}}\left(\frac{1}{Q}-\frac{1}{Q_{0}}\right)\right] ; \quad \frac{A_{6}\left(Q_{0}\right)}{\left(Q^{2}\right)^{3}} \exp \left[-\frac{8}{5 \lambda_{6}}\left(\frac{1}{Q}-\frac{1}{Q_{0}}\right)\right] \text {. }
$$

Here the term 'naive' refers to vacuum saturation at the level of local operators and subsequent delocalization of the scalar 2-point functions (see also ref. [20]). Eq. (10) implies that conventional, dimension 4 and 6 power corrections are drastically altered if $Q$ is of the order of $\lambda^{-1}$ or less. Despite the fact that the expression for dimension 6 in eq. (11) heavily relies on the vacuum saturation hypothesis it may feature a general trend of decreasing (effective) correlation lengths in higher mass dimensions (ultimately, only the lattice can decide on the truth of this assertion).

Let us extrapolate the behavior of eq. (11) to non-perturbative corrections of arbitrary dimension $d$. If the running with $Q$ essentially can be expressed as

$$
\frac{A_{d}\left(Q_{0}\right)}{Q^{d}} \exp \left[-\lambda_{d}^{-1}\left(\frac{1}{Q}-\frac{1}{Q_{0}}\right)\right]
$$

where $\lambda_{d}$ denotes an effective correlation length, then there is a single maximum at momentum $Q=\left(d \lambda_{d}\right)^{-1}$ with value

$$
P_{d} \equiv\left(\Lambda_{d} \lambda_{d} \exp [-1] d\right)^{d} \exp \left[\frac{\lambda_{d}^{-1}}{Q_{0}}\right]
$$

where $\Lambda_{d} \equiv\left(A_{d}\left(Q_{0}\right)\right)^{(1 / d)}$. So a non-perturbative correction at fixed $d$ vanishes asymptotically for both, $Q \rightarrow \infty$ and $Q \rightarrow 0$, which is in stark contrast to the 
conventional picture. This, however, does by no means imply that we approach asymptotic freedom at low momenta since higher and higher mass dimensions may contribute their maxima at lower and lower $Q$. One possibility for this to happen is the following: Let $\lambda_{d}$ fall off as $\lambda_{d}=\lambda / d^{(1-\varepsilon)},(1>\varepsilon>0)$, then the positions of the maxima still decrease as $Q=\lambda^{-1} d^{-\varepsilon}$. The values of these maxima behave as

$$
P_{d}=\left(\Lambda_{d} \lambda d^{\varepsilon} \exp \left[-1+\frac{d^{-\varepsilon}}{\lambda Q_{0}}\right]\right)^{d}
$$

In particular, assuming that $\Lambda_{d} \equiv \Lambda_{Q C D}$ as it is suggested by conventional OPE's, the critical dimension $d_{c}$ from which on maxima explode is given implicitely as a function of $\lambda, \varepsilon, \Lambda_{Q C D}$, and $Q_{0}$ by

$$
\Lambda_{Q C D} \lambda d^{\varepsilon} \exp \left[-1+\frac{d^{-\varepsilon}}{\lambda Q_{0}}\right]=1 .
$$

Truncating the OPE at some $d_{t}<d_{c}$ would mean that physics below the corresponding $Q$ is not described. A truncation at $d_{t} \geq d_{c}$ does not make sense since due to the rapid increase of the contributions at $d \geq d_{c}$ we are surely at orders where the expansion does not approximate (asymptotic expansion). Let us give some numerical examples. For $\varepsilon=0.5, \Lambda_{Q C D}=0.4 \mathrm{GeV}$ we calculate (the rounded) $d_{c}$ as a function of $\lambda$

$$
\begin{aligned}
& \lambda=3 \mathrm{GeV}^{-1} \rightarrow d_{c}=4 ; \quad \lambda=2 \mathrm{GeV}^{-1} \quad \rightarrow \quad d_{c}=10 \\
& \lambda=1 \mathrm{GeV}^{-1} \rightarrow d_{c}=40 ; \quad \lambda=0.5 \mathrm{GeV}^{-1} \quad \rightarrow \quad d_{c}=158
\end{aligned}
$$

Lattice measurements with $N_{F}=4$ staggered fermions of mass $a \cdot m_{q}=0.01$ and lattice resolution $Q_{0}=a^{-1} \sim 2 \mathrm{GeV}$ suggest that fermionic and gluonic correlation lengths corresponding to scalar correlation functions are $\sim 3.2 \mathrm{GeV}^{-1}$ and $\sim 1.7$ $\mathrm{GeV}^{-1}$, respectively [14]. So for dimension 4 and assuming (naive) vacuum saturation also for dimension 6 this does not pose a problem for the conventional sum rule analysis of light quark correlators (at $Q_{0}=a^{-1} \sim 2 \mathrm{GeV} A_{\bar{q} q}\left(Q_{0}\right)$ as well as $A_{F^{2}}\left(Q_{0}\right)$ seem to be compatible with their phenomenological values at $\mu \sim 1 \mathrm{GeV}$ [14]). However, (naive) vacuum saturation and/or the exclusion of higher mass dimensions do not lead to a realistic behavior of the spectral functions which is shown in 20]. Still, going only to dimension 6, one obtains a much more realistic picture if the (effective) correlation length at this dimension is drastically decreased (by about 10 times!). This can be motivated by the small correlation length of the 2-point vector function on the one hand (naive vacuum saturation not applicable). On the other hand this short correlation length may cumulatively simulate higher mass dimensions. The tendency that dimension 6 contributions are overestimated in the conventional OPE of [2] for distances $>0.3 \mathrm{fm}$ can be extracted from the work of [22]. There, the euclidean position space $V \pm A$ correlators were calculated in a random instanton liquid model and compared to the ALEPH data [24] and the 
conventional OPE. Note, however, that fitting experimental data to a conventional OPE does violate local duality. We investigate the issue in [23].

In the modern literature there is supporting evidence in favor of large mass scales (as compared to $\Lambda_{Q C D} \sim 0.2-0.4 \mathrm{GeV}$ ) characterizing the QCD vacuum: Based on lattice data for the instanton size distribution it was stated in ref. Shuryak that the average size of instantons is much smaller $(\sim 1 / 3 \mathrm{fm})$ than their mean separation $(\sim 1 \mathrm{fm})$. Using maximal Abelian projection on the lattice to identify magnetic monopoles, the size of these defects, $r \sim 0.05 \mathrm{fm}$, was determined for an $\mathrm{SU}(2)$ pure gauge theory $(\beta \sim 2.5)$ in ref. [26]. To calibrate the lattice the $\mathrm{SU}(3)$ value of the string tension $\sigma=440 \mathrm{MeV}$ was used. It was found in [27] that a phenomenologically motivated tachyonic gluon mass can take values well above the $1 \mathrm{GeV}$ bound.

To summarize, we proposed to use non-local, non-perturbative information on the QCD vacuum (extendable to hadronic states) for a non-perturbative procedure to coarse grain the euclidean VEV's (or hadronic matrix elements) of local operators composed of fundamental fields down to resolutions where their relevance must be questioned. Implications for the low-energy behavior of the OPE in the euclidean were discussed. In particular, a possibility for the determination of the critical operator dimension, where the breakdown of the OPE sets in, was outlined.

\section{Acknowledgements}

The author would like to thank Uli Nierste for a stimulating conversation. Financial support from CERN's theory group during a research stay in June are gratefully acknowledged. The author is indebted to V. I. Zakharov for numerous useful discussions and valuable comments.

\section{References}

[1] H. Fritzsch, M. Gell-Mann, and H. Leutwyler, Phys. Lett. B 47, 365 (1973). D. J. Gross and F. Wilczek, Phys. Rev. D8, 3633 (1973). H. D. Politzer, Phys. Rev. Lett. 30, 1346 (1973).

[2] M. Shifman, A. Vainshtein, and V. Zakharov, Nucl. Phys. B147, 385 (1979).

[3] K. G. Wilson, Phys. Rev. 179, 1499 (1969).

[4] M. Shifman, hep-ph/0009131.

[5] I. Bigi and N. Uraltsev, hep-ph/0106346.

[6] M. Shifman, Theory of Preasymptotic Effects in Weak Inclusive Decays, in Proc. Workshop on Continuous Advances in QCD, ed. A. Smilga (World Scientific, Singapore, 1994) 
[7] M. Golterman and S. Peris, JHEP 0101, 028 (2001).

[8] M. Shifman, in Particles, Strings and Cosmology, eds. J. Bagger et al., World Scientific Singapore 1996, hep-ph/9505289.

B. Chibisov, R. D. Dikeman, M. Shifman, and N. Uraltsev, Int. J. Mod. Phys. bf A 12, 2075 (1997), hep-ph/9605465.

B. Blok, M. Shifman, and D. Zhang, Phys. Rev. D57, 2691 (1998); (E) D59, 019901 (1999).

[9] M. Beneke, G. Buchalla, C. Greub, A. Lenz, and U. Nierste, Phys. Lett. B 459, 631.

U. Nierste, hep-ph/0009203.

[10] M. Neubert, Lectures on the theory of non-leptonic B decays, hep-ph/0012204. M. Neubert, Phys. Rev. D49, 4623 (1994).

[11] I. Dunietz, R. Fleischer, and U. Nierste, Phys. Rev. D63, 114015 (2001).

[12] R. Hofmann, hep-ph/0103279, to app. in Phys. Rev. D.

[13] H. G. Dosch, Phys. Lett. B 190, 177 (1987).

H. G. Dosch and Yu. A. Simonov, Phys. Lett. B 205, 339 (1988).

[14] M. D'Elia, A. Di Giacomo, and E. Meggiolaro, Phys. Lett. B408, 315 (1997).

A. Di Giacomo, E. Meggiolaro, and H. Panagopoulos, Nucl. Phys. B483, 371 (1997).

M. D'Elia, A. Di Giacomo, E. Meggiolaro, Phys. Rev. D59, (1999) 054503.

G.S. Bali, N. Brambilla, and A. Vairo, Phys. Lett. B421, 265 (1998).

[15] A.E. Dorokhov, S.V. Esaibegian and S.V. Mikhailov, Phys. Rev. D56, 4062 (1997).

A.E. Dorokhov, S.V. Esaibegian, A.E. Maximov and S.V. Mikhailov, Eur.Phys.J. C13, 331-345 (2000).

A.E. Dorokhov, L. Tomio, Phys. Rev. D62, 014016 (2000).

[16] V. I. Chernyak and A. R. Zhitnitsky, Nucl. Phys. B201, 492 (1982);

B214 547(E) (1983); Phys. Rept. 212, 173 (1984).

[17] S.V. Mikhailov and A. V. Radyushkin, Phys. Rev. D45, (1992) 1754.

A.P. Bakulev and S.V. Mikhailov, Phys. Lett. B436, 351 (1998).

[18] S.A. Anikin and O.I. Zavialov, Ann. Phys. 116, 135 (1978).

S.A. Anikin, O.I. Zavialov, and N.I. Karchev, Theor. Mat. Fiz. 38, 291 (1979).

N.I. Karchev, Nucl. Phys. B211, 55 (1983).

[19] I.I. Balitsky and V.M. Braun, Nucl. Phys. B311, 541 (1988/89). 
[20] R. Hofmann, hep-ph/0109.

[21] H. G. Dosch, M. Eidemüller, and M. Jamin, Phys. Lett. B452, 379 (1999).

[22] T. Schäfer and E. V. Shuryak, Phys. Rev. Lett. 86, 3973 (2001).

[23] R. Hofmann, hep-ph/0109.

[24] R. Barate et al.[ALEPH Collaboration], Z. Phys. C76, 15 (1997);

Eur. Phys. J. C4, 409 (1998).

[25] E. V. Shuryak, hep-ph/9909458.

[26] V. G. Bornyakov et al., hep-lat/0103032.

[27] K. G. Chetyrkin, S. Narison, V. I. Zakharov, Nucl. Phys. B550, 353 (1999). 